\title{
Implicit difference approximation of the Galilei invariant fractional advection diffusion equation
}
Changming Chen ${ }^{1}$
F. $\mathrm{Liu}^{2}$
I. Turner ${ }^{3}$
V. $\operatorname{Anh}^{4}$

(Received 15 August 2006; revised 09 December 2007)

\begin{abstract}
A Galilei invariant fractional advection diffusion equation with initial-boundary conditions is considered. An implicit difference approximation for solving the Galilei invariant fractional advection diffusion equation is presented. We introduce a new Fourier method for analyzing the stability and convergence of the implicit difference approximation. Finally, some numerical examples are given. The numerical results are in good agreement with our theoretical analysis. This method and supporting theoretical techniques can also be extended to a larger class of fractional integro-differential equations.
\end{abstract}

See http://anziamj.austms.org.au/ojs/index.php/ANZIAMJ/article/view/83 for this article, (c) Austral. Mathematical Soc. 2007. Published December 31, 2007. ISSN 1446-8735 


\section{Contents}

1 Introduction

C776

2 An implicit difference approximation for the GI-FADE

C778

3 Stability of the IDA for the GI-FADE

C779

4 Convergence of the IDA for the GI-FADE

C782

5 Numerical results

C785

6 Conclusions

C787

References

C787

\section{Introduction}

There has been increasing interest in the description of physical phenomena exhibiting anomalous diffusion, that is, diffusion not accurately modeled by the usual advection diffusion equation. An extension of the continuous time random walk approach to anomalous diffusion leads to fractional advection-dispersion equations (FADE). These equations have been used to describe transport in amorphous semiconductors, spread of contaminants in underground water, relaxation in polymer systems, and tracer dynamics in polymer networks (Sokolov et al. [7]).

We consider the numerical solution of the following initial/boundary value problem of the Galilei invariant FADE (GI-FADE) (Metzler et al. [4])

$$
\begin{aligned}
\frac{\partial W(x, t)}{\partial t}+v \frac{\partial W(x, t)}{\partial x}= & { }_{0} D_{t}^{1-\gamma} K_{\gamma} \frac{\partial^{2} W(x, t)}{\partial x^{2}}+f(x, t), \\
& 0<t \leq T, \quad 0<x<L,
\end{aligned}
$$


where $0<\gamma<1, K_{\gamma}>0$ and $v>0$ are constants, the function $f(x, t)$ can be used to represent sources and sinks, and ${ }_{0} D_{t}^{1-\gamma} V(x, t)$ is the RiemannLiouille fractional derivative of order $1-\gamma$ defined by Podlubny [6]

$$
{ }_{0} D_{t}^{1-\gamma} V(x, t)=\frac{1}{\Gamma(\gamma)} \frac{\partial}{\partial t} \int_{0}^{t} \frac{V(x, \eta)}{(t-\eta)^{1-\gamma}} d \eta \text {. }
$$

We impose on (1) the following initial and nonhomogeneous Dirichlet boundary conditions:

$$
\begin{aligned}
& W(x, 0)=\varphi(x), \quad 0 \leq x \leq L, \\
& W(0, t)=\phi(t), \quad 0<t \leq T \\
& W(L, t)=\psi(t), \quad 0<t \leq T
\end{aligned}
$$

The FADE has been recently treated by Liu et al. [2]. Yuste and Acedo [8] proposed an explicit finite difference method and a new von Neumann-type stability analysis for the fractional subdiffusion equation, that is, the GI-FADE without the advection term. However, they did not give the convergence analysis and pointed out the difficulty of this task when implicit methods are considered. Langlands and Henry [1] also investigated this problem and proposed an implicit numerical scheme (L1 approximation), and discussed its accuracy and stability. However, the global accuracy of the implicit numerical scheme was not derived and it seems that the unconditional stability for all $\gamma$ in the range $0<\gamma \leq 1$ has not been established. The main purpose of this article is to address these issues for the GI-FADE. We analyze the problem via a Fourier method.

Section 2 proposes an implicit difference approximation (IDA) for GI-FADE. The stability and convergence of the IDA are discussed using Fourier analysis in Sections 3 and 4, respectively. Finally, some numerical results will be given to evaluate the accuracy of the method. 


\section{An implicit difference approximation for the GI-FADE}

This section proposes an implicit difference approximation for solving the GI-FADE (1) with the initial and boundary conditions (3)-(5).

We take an equally spaced mesh of $M$ points for the spatial domain $0 \leq x \leq L$, and $N$ constant time steps for the temporal domain. We denote the spatial grid points and the temporal grid points by $x_{j}=j h$, $j=0,1, \ldots, M, t_{k}=k \tau, k=0,1, \ldots, N$, respectively, where the grid spacing is simply $h=L / M$ in the spatial domain and $\tau=T / N$ in the time domain.

Meerschaert et al. [5] showed that using the usual Grünwald formula to discretize the one dimensional fractional diffusion equation results in an unstable finite difference scheme. Thus, we start here with a right-shifted Grünwald approximation to the fractional derivative, which for $0<\gamma \leq 1$ is

$$
{ }_{0} D_{t}^{1-\gamma} V(x, t)=\tau^{\gamma-1} \sum_{l=0}^{[t / \tau]} w_{l}^{1-\gamma} V(x, t-l \tau)+O\left(\tau^{p}\right) .
$$

This formula is not unique because there are many different valid choices for $w_{l}^{1-\gamma}$ that lead to approximations of different order $p$ (Ch. Lubich [3]).

We now present the following IDA for the initial/boundary value problem of the GI-FADE (1)-(5):

$$
\begin{aligned}
& \frac{W_{j}^{k}-W_{j}^{k-1}}{\tau}+v \frac{W_{j+1}^{k}-W_{j-1}^{k}}{2 h} \\
= & \tau^{\gamma-1} K_{\gamma} \sum_{m=0}^{k} w_{m}^{1-\gamma} \frac{W_{j-1}^{k-m}-2 W_{j}^{k-m}+W_{j+1}^{k-m}}{h^{2}}+f_{j}^{k}, \\
& j=1,2, \ldots, M-1, \quad k=1,2, \ldots, N,
\end{aligned}
$$




$$
\begin{aligned}
& \qquad \begin{array}{l}
W_{0}^{k}=\phi\left(t_{k}\right), \quad W_{M}^{k}=\psi\left(t_{k}\right), \quad k=1,2, \ldots, N, \\
W_{j}^{0}=\varphi\left(x_{j}\right), \quad j=0,1, \ldots, M, \\
\text { where } \quad w_{m}^{1-\gamma}=(-1)^{m}\left(\begin{array}{c}
1-\gamma \\
m
\end{array}\right), \quad m=0,1, \ldots, k,
\end{array}
\end{aligned}
$$

and $f_{j}^{k} \equiv f\left(x_{j}, t_{k}\right)$. We take $p=1$. These coefficients can be evaluated as follows (Yuste et al. [8])

$$
w_{0}^{\alpha}=1, \quad w_{m}^{\alpha}=(-1)^{m} \frac{\alpha(\alpha-1) \cdots(\alpha-m+1)}{m !}, \quad m=1,2, \ldots .
$$

\section{Stability of the IDA for the GI-FADE}

This section analyzes the stability of the IDA using Fourier analysis. Firstly, we rewrite (7) as

$$
\begin{aligned}
W_{j}^{k}= & W_{j}^{k-1}+\mu_{2} \sum_{m=0}^{k} w_{m}^{1-\gamma}\left(W_{j-1}^{k-m}-2 W_{j}^{k-m}+W_{j+1}^{k-m}\right) \\
& -\mu_{1} \frac{W_{j+1}^{k}-W_{j-1}^{k}}{2}+\tau f_{j}^{k}, \quad j=1,2, \ldots, M-1, \quad k=1,2, \ldots, N
\end{aligned}
$$

where $\mu_{1}=v \tau / h$ and $\mu_{2}=K_{\gamma} \tau^{\gamma} / h^{2}$. The roundoff error $\rho_{j}^{k}$ of the solution for the IDA (7)-(9) satisfy the difference equation

$$
\begin{array}{r}
\rho_{j}^{k}=\rho_{j}^{k-1}-\mu_{1} \frac{\rho_{j+1}^{k}-\rho_{j-1}^{k}}{2}+\mu_{2} \sum_{m=0}^{k} w_{m}^{1-\gamma}\left(\rho_{j-1}^{k-m}-2 \rho_{j}^{k-m}+\rho_{j+1}^{k-m}\right), \\
j=1,2, \ldots, M-1, \quad k=1,2, \ldots, N .
\end{array}
$$

We now define the grid functions:

$$
\rho^{k}(x)=\left\{\begin{aligned}
\rho_{j}^{k}, & \text { when } x_{j}-\frac{h}{2}<x \leq x_{j}+\frac{h}{2}, \quad j=1,2, \ldots, M-1, \\
0, & \text { when } 0 \leq x \leq \frac{h}{2} \text { or } \quad L-\frac{h}{2}<x \leq L,
\end{aligned}\right.
$$


and note that $\rho^{k}(x)$ extended in a Fourier series as

$$
\rho^{k}(x)=\sum_{l=-\infty}^{\infty} d_{k}(l) e^{i 2 \pi l x / L}, \quad k=1,2, \ldots, N
$$

where $d_{k}(l)=\frac{1}{L} \int_{0}^{L} \rho^{k}(x) e^{-i 2 \pi l x / L} d x, i=\sqrt{-1}$. We let

$$
\rho^{k}=\left[\rho_{1}^{k}, \rho_{2}^{k}, \ldots, \rho_{M-1}^{k}\right]^{T}
$$

and introduce the following norm:

$$
\left\|\rho^{k}\right\|_{2}=\left(\sum_{j=1}^{M-1} h\left|\rho_{j}^{k}\right|^{2}\right)^{1 / 2}=\left[\int_{0}^{L}\left|\rho^{k}(x)\right|^{2} d x\right]^{1 / 2} .
$$

Based on the Parseval equality:

$$
\int_{0}^{L}\left|\rho^{k}(x)\right|^{2} d x=\sum_{l=-\infty}^{\infty}\left|d_{k}(l)\right|^{2}
$$

we have

$$
\left\|\rho^{k}\right\|_{2}^{2}=\sum_{l=-\infty}^{\infty}\left|d_{k}(l)\right|^{2}
$$

We now assume that the solution of equation (12) has the following form:

$$
\rho_{j}^{k}=d_{k} e^{i \sigma j h},
$$

where $\sigma=2 \pi l / L$. Substituting the above expression into (12), we obtain

$$
d_{k}=d_{k-1}-i \mu_{1} \sin (\sigma h) d_{k}-4 \mu_{2} \sin ^{2} \frac{\sigma h}{2} \sum_{m=0}^{k} w_{m}^{1-\gamma} d_{k-m}, \quad k=1,2, \ldots, N .
$$


Lemma 1 The coefficients $w_{m}^{1-\gamma}(m=0,1, \ldots)$ satisfy
1. $w_{0}^{1-\gamma}=1 ; \quad w_{1}^{1-\gamma}=\gamma-1 ; \quad w_{m}^{1-\gamma}<0, m=1,2, \ldots ;$
2. $\sum_{m=0}^{\infty} w_{m}^{1-\gamma}=1$; for all $n \in N,-\sum_{m=1}^{n} w_{m}^{1-\gamma}<1$.

Zhuang et al. [9] gave the proof.

Applying Lemma 1, equation (15) is rewritten as

$$
d_{k}=\frac{1+(1-\gamma) \bar{\mu}}{1+\bar{\mu}+i \tilde{\mu}} d_{k-1}-\frac{\bar{\mu}}{1+\bar{\mu}+i \tilde{\mu}} \sum_{m=2}^{\infty} w_{m}^{1-\gamma} d_{k-m}, \quad k=1,2, \ldots, N
$$

where $\bar{\mu}=4 \mu_{2} \sin ^{2} \frac{\sigma h}{2} \geq 0$ and $\tilde{\mu}=\mu_{1} \sin \sigma h$.

Lemma 2 Assuming that $d_{k}(k=1,2 \ldots, N)$ is the solution of equation (16), then

$$
\left|d_{k}\right| \leq\left|d_{0}\right|, \quad k=1,2, \ldots, N .
$$

Proof: Mathematical induction proves this result.

Theorem 3 The IDA (7)-(9) for the GI-FADE (1)-(5) is unconditionally stable.

Proof: Apply Lemma 2 and noting (13), we have

$$
\left\|\rho^{k}\right\|_{2} \leq\left\|\rho^{0}\right\|_{2}, \quad k=1,2, \ldots, N,
$$

which implies that the IDA (7)-(9) for the GI-FADE (1)-(5) is unconditionally stable. 


\section{Convergence of the IDA for the GI-FADE}

This section discusses the convergence of the IDA. Let

$$
\begin{aligned}
R_{j}^{k}= & \frac{W\left(x_{j}, t_{k}\right)-W\left(x_{j}, t_{k-1}\right)}{\tau}+v \frac{W\left(x_{j+1}, t_{k}\right)-W\left(x_{j-1}, t_{k}\right)}{2 h} \\
& -\tau^{\gamma-1} K_{\gamma} \sum_{m=0}^{k} w_{m}^{1-\gamma} \frac{W\left(x_{j-1}, t_{k-m}\right)-2 W\left(x_{j}, t_{k-m}\right)+W\left(x_{j+1}, t_{k-m}\right)}{h^{2}} \\
& -f\left(x_{j}, t_{k}\right), \quad j=1,2, \ldots, M-1, \quad k=1,2, \ldots, N .
\end{aligned}
$$

The following lemma holds.

Lemma $4 \tau^{\gamma-1} \sum_{m=0}^{k} w_{m}^{1-\gamma}=\frac{1}{\Gamma(\gamma)}+O(\tau)$.

Applying the Taylor expansions, (6) and Lemma 4, we obtain

$$
\left|R_{j}^{k}\right| \leq C_{1}\left(\tau+h^{2}\right), \quad j=1,2, \ldots, M-1, \quad k=1,2, \ldots, N,
$$

where $C_{1}$ is a positive constant. From (19), we have

$$
\begin{aligned}
& W\left(x_{j}, t_{k}\right)=W\left(x_{j}, t_{k-1}\right)-\mu_{1} \frac{W\left(x_{j+1}, t_{k}\right)-W\left(x_{j-1}, t_{k}\right)}{2} \\
& +\mu_{2} \sum_{m=0}^{k} w_{m}^{1-\gamma}\left[W\left(x_{j-1}, t_{k-m}\right)-2 W\left(x_{j}, t_{k-m}\right)+W\left(x_{j+1}, t_{k-m}\right)\right] \\
& +\tau f\left(x_{j}, t_{k}\right)+\tau R_{j}^{k}, \quad j=1,2, \ldots, M-1, \quad k=1,2, \ldots, N .
\end{aligned}
$$

Subtracting (11) from (21), we obtain

$$
\begin{gathered}
e_{j}^{k}=e_{j}^{k-1}-\mu_{1} \frac{e_{j+1}^{k}-e_{j-1}^{k}}{2}+\mu_{2} \sum_{m=0}^{k} w_{m}^{1-\gamma}\left(e_{j-1}^{k-m}-2 e_{j}^{k-m}+e_{j+1}^{k-m}\right) \\
+\tau R_{j}^{k}, \quad j=1,2, \ldots, M-1, \quad k=1,2, \ldots, N
\end{gathered}
$$


where $e_{j}^{k}=W\left(x_{j}, t_{k}\right)-W_{j}^{k}, j=0,1, \ldots, M, k=0,1, \ldots, N$.

We now analyze the convergence of the IDA by using Fourier analysis. We define the grid functions for $k=0,1, \ldots, N$ as

$$
e^{k}(x)=\left\{\begin{aligned}
e_{j}^{k}, & \text { when } x_{j}-\frac{h}{2}<x \leq x_{j}+\frac{h}{2}, \quad j=1,2, \ldots, M-1, \\
0, & \text { when } 0 \leq x \leq \frac{h}{2} \quad \text { or } \quad L-\frac{h}{2}<x \leq L,
\end{aligned}\right.
$$

and

$$
R^{k}(x)=\left\{\begin{aligned}
R_{j}^{k}, & \text { when } x_{j}-\frac{h}{2}<x \leq x_{j}+\frac{h}{2}, \quad j=1,2, \ldots, M-1, \\
0, & \text { when } 0 \leq x \leq \frac{h}{2} \quad \text { or } \quad L-\frac{h}{2}<x \leq L,
\end{aligned}\right.
$$

respectively. Thus, $e^{k}(x)$ and $R^{k}(x)$ have extended Fourier series expansions

$$
e^{k}(x)=\sum_{l=-\infty}^{\infty} \xi_{k}(l) e^{i 2 \pi l x / L}, \quad k=0,1, \ldots, N
$$

and

$$
R^{k}(x)=\sum_{l=-\infty}^{\infty} \eta_{k}(l) e^{i 2 \pi l x / L}, \quad k=1,2, \ldots, N
$$

respectively, where

$$
\xi_{k}(l)=\frac{1}{L} \int_{0}^{L} e^{k}(x) e^{-i 2 \pi l x / L} d x, \quad \eta_{k}(l)=\frac{1}{L} \int_{0}^{L} R^{k}(x) e^{-i 2 \pi l x / L} d x .
$$

We now let

$$
e^{k}=\left[e_{1}^{k}, e_{2}^{k}, \ldots, e_{M-1}^{k}\right]^{T}, \quad k=0,1, \ldots, N
$$

and

$$
R^{k}=\left[R_{1}^{k}, R_{2}^{k}, \ldots, R_{M-1}^{k}\right]^{T},
$$

and introduce the following norms:

$$
\left\|e^{k}\right\|_{2}=\left(\sum_{j=1}^{M-1} h\left|e_{j}^{k}\right|^{2}\right)^{1 / 2}=\left[\int_{0}^{L}\left|e^{k}(x)\right|^{2} d x\right]^{1 / 2}, \quad k=0,1, \ldots, N
$$


and

$$
\left\|R^{k}\right\|_{2}=\left(\sum_{j=1}^{M-1} h\left|R_{j}^{k}\right|^{2}\right)^{1 / 2}=\left[\int_{0}^{L}\left|R^{k}(x)\right|^{2} d x\right]^{1 / 2}, \quad k=1,2, \ldots, N,
$$

respectively. Using Parseval's equality:

$$
\begin{aligned}
& \int_{0}^{L}\left|e^{k}(x)\right|^{2} d x=\sum_{l=-\infty}^{\infty}\left|\xi_{k}(l)\right|^{2}, \quad k=0,1, \ldots, N, \\
& \int_{0}^{L}\left|R^{k}(x)\right|^{2} d x=\sum_{l=-\infty}^{\infty}\left|\eta_{k}(l)\right|^{2}, \quad k=1,2, \ldots, N, \\
& \left\|e_{k}\right\|_{2}^{2}=\sum_{l=-\infty}^{\infty}\left|\xi_{k}(l)\right|^{2}, \quad k=0,1, \ldots, N, \\
& \left\|R_{k}\right\|_{2}^{2}=\sum_{l=-\infty}^{\infty}\left|\eta_{k}(l)\right|^{2}, \quad k=1,2, \ldots, N,
\end{aligned}
$$

respectively. From the above analysis, we now suppose that

$$
e_{j}^{k}=\xi_{k} e^{i \sigma j h}
$$

and

$$
R_{j}^{k}=\eta_{k} e^{i \sigma j h},
$$

where $\sigma=2 \pi l / L$. Substituting (36) and (37) into (22), we obtain

$$
\begin{aligned}
\xi_{k}= & \xi_{k-1}-i \mu_{1} \sin \sigma h \cdot \xi_{k}-4 \mu_{2} \sin ^{2} \frac{\sigma h}{2} \sum_{m=0}^{k} w_{m}^{1-\gamma} \xi_{k-m}+\tau \eta_{k}, \\
& k=1,2, \ldots, N
\end{aligned}
$$

Applying Lemma 1, rewrite equation (38) as

$$
\xi_{k}=\frac{1+(1-r) \bar{\mu}}{1+\bar{\mu}+i \tilde{\mu}} \xi_{k-1}-\frac{\bar{\mu}}{1+\bar{\mu}+i \tilde{\mu}} \sum_{m=2}^{k} w_{m}^{1-\gamma} \xi_{k-m}
$$




$$
+\frac{1}{1+\bar{\mu}+i \tilde{\mu}} \tau \eta_{k}, \quad k=1,2, \ldots, N
$$

where $\bar{\mu}=4 \mu_{2} \sin ^{2}(\sigma h / 2) \geq 0$ and $\tilde{\mu}=\mu_{1} \sin \sigma h$.

Similar to the proof of Lemma 2, we also obtain the following lemma.

Lemma 5 Supposing that $\xi_{k}(k=1,2 \ldots, N)$ is the solution of equation (39), then there is a positive constant $C_{2}$ such that

$$
\left|\xi_{k}\right| \leq C_{2} k \tau\left|\eta_{1}\right|, \quad k=1,2, \ldots, N .
$$

Theorem 6 The IDA (7)-(9) for the GI-FADE (1)-(5) is convergent, and the convergence order is $O\left(\tau+h^{2}\right)$.

Proof: Noting (34) and (35), and applying Lemma 5, we obtain the result

$$
\left\|e^{k}\right\|_{2} \leq C_{2} k \tau\left\|R^{1}\right\|_{2} \leq C_{1} C_{2} k \tau \sqrt{L}\left(\tau+h^{2}\right) .
$$

As $k \tau \leq T$,

$$
\left\|e^{k}\right\|_{2} \leq C\left(\tau+h^{2}\right),
$$

where $C=C_{1} C_{2} T \sqrt{L}$. The result then follows.

\section{$5 \quad$ Numerical results}

This section gives a numerical example that confirms our theoretical analysis. We consider the initial-boundary value problem of the GI-FADE with a nonhomogeneous source term:

$$
\frac{\partial W(x, t)}{\partial t}+\frac{\partial W(x, t)}{\partial x}={ }_{0} D_{t}^{1-\gamma} \frac{\partial^{2} W(x, t)}{\partial x^{2}}
$$


TABLE 1: The maximum error $E_{\infty}$.

\begin{tabular}{ccccc}
\hline$\tau$ & $h$ & $\gamma=0.4$ & $\gamma=0.5$ & $\gamma=0.6$ \\
\hline$\frac{1}{64}$ & $\frac{1}{8}$ & $9.7 \mathrm{E}-04$ & $1.3 \mathrm{E}-03$ & $1.6 \mathrm{E}-03$ \\
$\frac{1}{1024}$ & $\frac{1}{32}$ & $1.4 \mathrm{E}-04$ & $9.0 \mathrm{E}-05$ & $1.9 \mathrm{E}-04$ \\
\hline
\end{tabular}

$$
\begin{aligned}
& +e^{x}\left[(1+\gamma) t^{\gamma}+t^{1+\gamma}-\frac{\Gamma(2+\gamma)}{\Gamma(1+2 \gamma)} t^{2 \gamma}\right], \quad 0<t \leq 1, \quad 0<x<1 \\
& W(0, t)=t^{1+\gamma}, \quad W(1, t)=e t^{1+\gamma}, \quad 0<t \leq 1 \\
& W(x, 0)=0, \quad 0 \leq x \leq 1
\end{aligned}
$$

The exact solution of the problem (43)-(45) is

$$
W(x, t)=e^{x} t^{1+\gamma} .
$$

The maximum error of the exact and numerical solutions is defined as

$$
E_{\infty}=\max _{0 \leq j \leq M} \max _{0 \leq k \leq N}\left|W\left(x_{j}, t_{k}\right)-W_{j}^{k}\right| .
$$

Table 1 shows the maximum error at all mesh points for different $\gamma$ using $\tau=\frac{1}{64}, h=\frac{1}{8}$ and $\tau=\frac{1}{1024}, h=\frac{1}{32}$ respectively, and the effect of $\tau$ and $h$. Physical conditions impose the range $0<\gamma<1$. In this example we select three typical values $\gamma=0.4,0.5$ and 0.6 in this range. Our analysis indicates a convergence order of $O\left(\tau+h^{2}\right)$ for small $\tau$ and $h^{2}$ for the IDA scheme. The small values $\tau=1 / 64,1 / 1024$ and $h=1 / 8,1 / 32$ have been used in this example.

Table 1 indeed indicates that the maximum error is $O\left(\tau+h^{2}\right)$ and IDA is unconditionally stable. These numerical results are in good agreement with our theoretical analysis. 


\section{Conclusions}

Numerical treatment of fractional subdiffusion equations is known to be difficult. We consider the Galilei invariant fractional advection diffusion equation which covers fractional subdiffusion as a special case. We developed an implicit difference approximation for solving the GI-FADE. We introduced a Fourier method to successfully analyze the stability and convergence of the IDA. We proved that the IDA is unconditionally stable and convergent. This method and supporting theoretical techniques can also be extended to a larger class of fractional integro-differential equations.

Acknowledgements: This research has been supported by the National Natural Science Foundation of China grant 10271098, and the Australian Research Council grant LP0348653. The authors thank the referees for their suggestions to improve the paper.

\section{References}

[1] T. A. M. Langlands and B. I. Henry, The accuracy and stability of an implicit solution method for the fractional diffusion equation, J. Comp. Phys., 205 (2005), 719-736. doi:10.1016/j.jcp.2004.11.025 C777

[2] F. Liu, V. Anh, I. Turner, Numerical Solution of the Space Fractional Fokker-Planck Equation, J. Comp. Appl. Math., 166 (2004), 209-219. doi:10.1016/j.cam.2003.09.028 C777

[3] Ch. Lubich, Discretized fractional calculus, SIAM J. Math. Anal., 17 (1986), 704-719. C778 
[4] R. Metzler and J. Klafter, The random walk's guide to anomalous diffusion: a fractional dynamics approach, Phys. Reports, 339,(2000), 1-77. doi:10.1016/S0370-1573(00)00070-3 C776

[5] M. Meerschaert, C. Tadjeran, Finite difference approximations for fractional advection-dispersion flow equations, J. Comp. Appl. Math., 172 (2004), 65-77. doi:10.1016/j.cam.2004.01.033 C778

[6] I. Podlubny, Fractional Differential Equations, Academic Press, New York, 1999. http://people.tuke.sk/igor.podlubny/fde.html C777

[7] I. M. Sokolov, J. Klafter and A. Blumen, Fractional Kinetics, Physics Today, 55(11) (2002) 48-54. C776

[8] S. B. Yuste and L. Acedo, An explicit finite difference method and a new Von Neumann-type stability analysis for fractional diffusion equations, SIAM J. Numer. Anal., 42(5) (2005), 1862-1874. C777, C779

[9] P. Zhuang and F. Liu, Implicit difference approximation for the time fractional diffusion equation, J. Appl. Math. Comp., 22(3) (2006) 87-99.

http://jamc.net/contents/table_contents_view.php?idx=580 C781 


\section{Author addresses}

1. Changming Chen, School of Mathematical Sciences, Xiamen University, Xiamen 361005, China.

mailto: cmchen@xmu.edu.cn

2. F. Liu, School of Mathematical Sciences, South China University of Technology, Guangzhou 510640, CHINA.

mailto:f.liu@qut.edu.au, fwliu@xmu.edu.cn

3. I. Turner, School of Mathematical Sciences, Queensland University of Technology, Queensland 4001, Australia.

mailto:i.turner@qut.edu . au

4. V. Anh, School of Mathematical Sciences, Queensland University of Technology, Queensland 4001, Australia.

mailto:v. anh@qut.edu.au 